\title{
Processing of sequential tactile patterns: Effects of a neutral stimulus
}

\author{
JAMES C. CRAIG \\ Indiana University, Bloomington, Indiana
}

\begin{abstract}
The perception of a target pattern may be interfered with by the presentation of a nontarget pattern in close temporal and spatial proximity with it. The results from previous studies suggested that much of this interference is the result not of masking but of response competition, subjects responding with the nontarget instead of the target. Using a 4-to-2 paradigm in which four target patterns are mapped onto two responses, it was shown that neutral patterns (i.e., patterns with no responses associated with them) produce considerable interference. The amount of interference is less than that produced by patterns associated with incorrect responses but greater than that produced by patterns associated with correct responses. The amount of interference produced by neutral patterns did not vary as a function of the form of the neutral pattern (Experiments 1 and 2); however, the amount of interference did depend on the degree and nature of the similarity between the neutral and target patterns (Experiments 3 and 4). The results indicate that recent studies have underestimated the amount of interference due to masking and overestimated the amount due to response competition. Response competition may either hinder or help target categorization depending on the nontarget pattern.
\end{abstract}

The processing of tactual information generally involves the reception of sequences of spatial patterns, arriving at both single and multiple sites on the skin. A number of studies have examined the stimulus factors that affect the ability of subjects to attend to multiple sites of stimulation (Lederman \& Klatzky, 1997; Sathian \& Burton, 1991; Whang, Burton, \& Shulman, 1991) and the processing of patterns arriving sequentially at the same site. The focus of the present study was on this latter situation, which is encountered in reading braille and with the Optacon (Bliss, Katcher, Rogers, \& Shepard, 1970; Foulke, 1991; Hill, 1974) and in object recognition (Lederman \& Klatzky, 1993). It has been known for some time that the identification of a tactile pattern (a target) may be interfered with by the presentation of a second pattern (a nontarget) at the same location as the target. A number of studies have examined the nature of this interference (Cholewiak \& Craig, 1984; Craig, 1982; Schindler \& Knapp, 1976). Two factors have been singled out as important contributors to the interference produced by a nontarget pattern: masking and response competition (Craig, 1995; Craig \& Evans, 1995; Craig \& Qian, 1997).

Masking is generally considered to reduce target identification by altering the initial representation of the target pattern. One way the representation is altered is by displacing the apparent location of features of the target (Craig, 1989). A second, and probably more potent, way is

This research was supported by National Institutes of Health Grant DC00095, National Institute on Deafness and Other Communication Disorders. The author thanks Roger Rhodes for his assistance in these experiments. Correspondence should be addressed to J. C. Craig, Department of Psychology, Indiana University, Bloomington, IN 47405 (e-mail: craigi@indiana.edu). through temporal integration (Craig, 1996; Evans, 1987; Evans \& Craig, 1986; Mahar \& Mackenzie, 1993) in which the target and nontarget patterns form a single composite pattern. This composite representation may make it difficult for the subject to extract the features of the target pattern and identify it. In response competition, the target and nontarget are processed to the point of evoking a response, and the subject errs by selecting the response associated with the nontarget. This sort of error is thought to occur at a stage of processing later than masking.

Evidence that interference is due at least in part to response competition comes from a paradigm that has been used in studies of visual selective attention (Eriksen \& Hoffman, 1973). This paradigm has been referred to as the 4-to-2 paradigm because subjects are trained to categorize a set of four patterns by placing each pattern into one of two categories. In several recent studies and in the present study, this paradigm was used to study the processing of patterns presented sequentially to the same location. Using the 4-to-2 paradigm with tactile patterns, subjects are presented a target pattern and a nontarget pattern on each trial. The nontarget pattern is selected from the same set of four patterns as the target pattern. By the random pairing of target and nontarget patterns, three types of trials are created: stimulus compatible (SC), response compatible (RC), and response incompatible (RI). On SC trials, the target and nontarget are identical. One would expect to see little interference here, and little is seen. On $\mathrm{RC}$ trials, the target and nontarget are physically different from one another but are associated with the same response. One would expect to see some interference, possibly due to masking, and, in fact, some interference is seen. On RI trials, the target and nontarget are physically different and associated with different responses. Here, one 
would expect to see interference due to masking and additional interference due to response competition-that is, subjects responding incorrectly with the nontarget (Craig, 1995; Craig \& Evans, 1995; Craig \& Qian, 1997). More details of the paradigm are presented in Experiment 1 and in Tables $1 \mathrm{~A}$ and $1 \mathrm{~B}$.

In assessing the mechanisms involved in interference, performance levels on RC trials are critical for two reasons. First, the difference between performance on RC and SC trials has been attributed to masking - that is, interference at a fairly early stage of processing. Second, the difference between RC and RI trials has been attributed to response competition. These differences are important for interpreting the results in the 4-to-2 paradigm. The present study focused on the possibility that performance on RC trials may not be solely the result of masking and on the need for an additional trial type in the 4to-2 paradigm, one involving neutral stimuli.

Performance on RI trials is thought to be low because subjects are responding with the nontarget, an incorrect response. By the same reasoning, performance on $\mathrm{RC}$ trials may be high because subjects are also responding with the nontarget (in this case, making a correct response). In other words, subjects can be correct on RC trials in two ways: either by responding with the target or by responding with the nontarget and being correct. A nontarget might be producing considerable masking, but only small declines in performance are observed because subjects are selecting the correct category by responding with the nontarget. The 4-to-2 paradigm, as it has been used so far with tactile stimuli, might underestimate the amount of interference due to masking. To see the extent to which masking might lower performance requires the use of a nontarget that evokes neither a correct nor an incorrect response - that is, a neutral nontarget. The present study used a 4-to-2 paradigm and examined the effects of neutral patterns on the perception of target patterns. The first aim of the present study was to see how target identification performance in the presence of neutral patterns compared with performance on RC and RI trials.

In previous studies of masking, a number of different types of interfering stimuli have been used, such as letterlike patterns, energy maskers, or dynamic maskers (Cholewiak \& Collins, 1988, 1997; Craig, 1982). These types of maskers can be considered neutral patterns because no responses were assigned to them, although it has also been suggested that some of these maskers contain features that might evoke target responses and interfere with target identification (Cholewiak \& Collins, 1997; Craig, 1982). Earlier studies were not carried out using a response competition paradigm. Thus, it is not possible to compare directly the amount of interference produced by nontarget patterns, such as energy and dynamic maskers (no response assigned to them), with nontarget patterns that evoke either correct or incorrect responses. A second aim of the present study was to examine the effectiveness of such maskers in a 4-to-2 paradigm.

The study consisted of four experiments. In Experiments 1 and 2 , the overall effects of neutral patterns on tar- get patterns were measured. In Experiments 3 and 4, the nature of the neutral patterns was examined. In these latter experiments, the similarity between neutral and target patterns was determined empirically. The aim was to see whether the similarity between the target and neutral patterns could be used to estimate the amount of interference.

\section{EXPERIMENT 1}

The purpose of Experiment 1 was to see what effect a neutral stimulus had on target identification and how the level of performance on neutral trials compared with that obtained on RC and RI trials. If RC performance were higher than performance with neutral patterns, it would indicate that the nontarget pattern on $\mathrm{RC}$ trials improves performance and, furthermore, that the performance on RC trials underestimates the amount of interference due to masking. Two types of neutral stimuli were used: an energy masker and a dynamic masker. These types of maskers, serving as nontargets, have the advantage that they are different from the target patterns in both the number of tactors activated and the shape of the patterns. They are unlikely to be confused with the simple target patterns used and thus unlikely to evoke either correct or incorrect responses.

\section{Method}

Subjects. The subjects in all four experiments received training in tactile pattern perception tasks prior to data collection. The subjects, ranging in age from 18 to 35 years, were undergraduate and graduate students at Indiana University who were paid an hourly rate for their participation. The subjects were selected from a group of subjects in the laboratory on the basis of their schedules and availability. In Experiment 1, 6 subjects were tested: 4 women and 2 men.

Apparatus. The apparatus has been used in previous studies of pattern identification (Craig, 1980; Evans \& Craig, 1992). The tactile display measured $1.1 \times 2.7 \mathrm{~cm}$ and fit against the subject's distal fingerpad. The array consisted of 144 tactors arranged in a 6 column $\times 24$ row array. In the present study, only the distal 18 rows of the display were used. Each tactor could be made to vibrate at 230 pulses/sec. The array was similar to that used in the Optacon, a reading aid for the blind (Bliss et al., 1970). A computer controlled both the tactile display and a visual monitor that was used to present instructions and feedback to the subjects. The subjects responded by means of a keyboard.

Stimuli. All patterns were presented for $26 \mathrm{msec}$. The four target patterns are shown in Figure 1. As indicated, the patterns were grouped into two categories labeled "I" and "II." Two types of patterns served as neutral patterns in the first set of measurements: an energy masker and a dynamic masker. Representations of these are also shown in Figure 1. The energy masker was generated by turning on all the tactors (108) that covered the distal portion of the array, the top 18 rows. The dynamic masker was generated by turning on a randomly selected set of 47 tactors for $4.3 \mathrm{msec}$, turning that set off, then turning on a second set of 47 tactors for $4.3 \mathrm{msec}$, turning that set off, and so forth, until six sets of tactors had been turned on and off for a total of $26 \mathrm{msec}$. This dynamic masker was designed to minimize the creation of contours that might be confused with the target pattern (Cholewiak \& Collins, 1997).

Procedure. The procedure was similar to that used in other studies of response competition and masking (Craig, 1995; Evans \& Craig, 1992). The subjects were trained to identify and place target patterns in one of several categories. Other patterns (nontargets) 


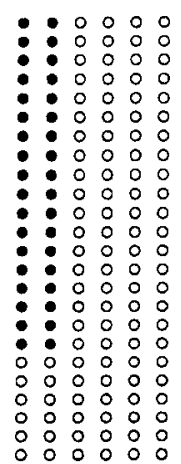

1

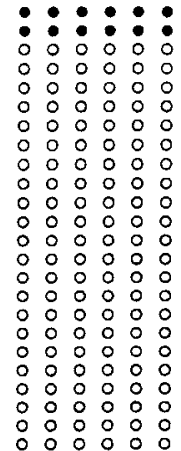

2

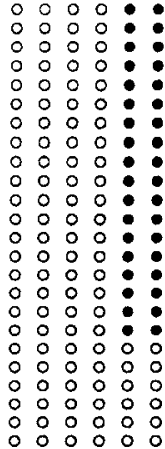

3 $\circ 0000$

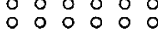

: $0: 000$

$\therefore: \circ \circ: \circ$

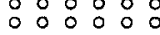

000

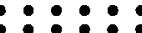

$0: 0: 0$

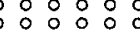

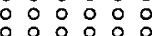

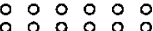

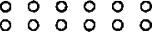

$\therefore$

$\begin{array}{llllll}0 & 0 & 0 & 0 & 0 & 0 \\ 0 & 0 & 0 & 0 & 0 & 0\end{array}$

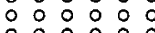

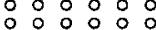

$0 \begin{array}{llllll}0 & 0 & 0 & 0 & 0 \\ 0 & 0 & 0 & 0 & 0 & 0\end{array}$

4
I

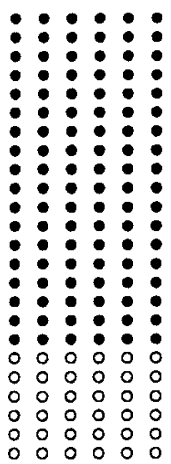

Energy Mask
II

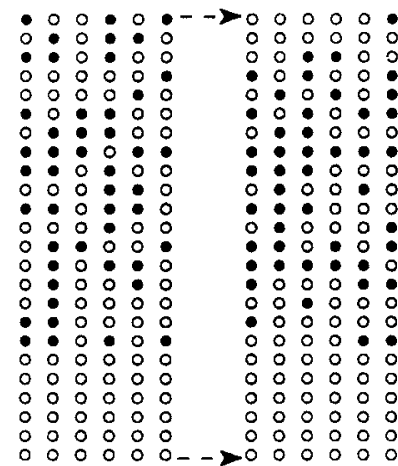

Frame 1 of 6 Frame 2 of 6

Figure 1. Representations of the four target patterns used in Experiment 1. Patterns 1 and 2 were placed in Category $I$; patterns 3 and 4 were placed in Category II. Representations of the two types of neutral patterns are also shown.

were presented to the same site in close temporal proximity to the target pattern.

The subjects placed their left hands on the table in front of them with their left index fingers extended and resting on the tactile array. The subjects responded with their right hands by means of a keyboard. As Table $1 \mathrm{~A}$ indicates, there were four target patterns (grouped into two categories) and two neutral patterns. On each trial, the subjects received two patterns presented sequentially to the same location: a target pattern and nontarget pattern selected at random. The nontarget patterns were selected either from the set of target patterns or from one of the two neutral patterns (energy or dynamic masker). The pairing of the target and nontarget patterns created four types of trials: SC, RC, RI, and neutral (see Table 1B).

Of the total trials, $16.5 \%$ were SC trials, $16.5 \%$ RC trials, $33 \%$ RI trials, and $33 \%$ were neutral trials. On neutral trials, half of the nontarget patterns were energy maskers and half dynamic maskers. Seven stimulus onset asynchronies (SOAs) were tested, -500 , $-300,-100,-26,+26,+100$, and $+300 \mathrm{msec}$, where negative values refer to conditions in which the nontarget preceded the target.
Each testing session consisted of eight blocks of 40 trials, including one block in which the target pattern was presented by itself. The same SOA was tested within a block of trials, and the subjects were instructed to identify either the first pattern or the second pattern (depending on whether the target pattern led or trailed the nontarget pattern during that block of trials). Within a session, the order of testing of each block of trials (each SOA) was determined randomly, with the proviso that the first block of trials was the block in which the target pattern was presented by itself. The subjects were tested for 17 sessions.

For all experiments, the driving voltage to the tactors was set at $36 \mathrm{~V}$, which produced a moderately intense, comfortable level of stimulation. At the beginning of each trial, a message was presented on the CRT telling the subjects to identify either the first or the second pattern. Following each trial a message appeared informing the subjects whether they were correct or, if incorrect, the category number corresponding to the correct response. The subjects initiated each trial by pressing a button on the keyboard. The target was presented $750 \mathrm{msec}$ later. Following the presentation of both the tar- 
Table 1A

Representation of Pattern Set in the 4-to-2 Paradigm

\begin{tabular}{cl}
\hline Pattern & Response \\
\hline $\mathrm{A}$ & 1 \\
$\mathrm{~B}$ & 1 \\
$\mathrm{C}$ & 2 \\
$\mathrm{D}$ & 2 \\
$\mathrm{~N}_{1}$ & None \\
$\mathrm{N}_{2}$ & None \\
\hline
\end{tabular}

Table 1B

Representation of Trial Types in the 4-to-2 Paradigm

\begin{tabular}{|c|c|c|c|}
\hline Trial Type & Target & + & Nontarget \\
\hline Stimulus Compatible & A & & $A$ \\
\hline Response Compatible & A & & B \\
\hline Response Incompatible & A & & $\mathrm{C}$ \\
\hline Neutral & A & & $\mathrm{N}_{1}$ \\
\hline
\end{tabular}

get and the nontarget, the subjects were allowed to respond. To eliminate any auditory cues, the subjects wore earplugs and earphones through which white noise was presented.

\section{Results and Discussion}

The neutral data were analyzed to determine whether the type of neutral nontarget (energy vs. dynamic) had any differential effect on the results. There was no significant effect due to type of neutral pattern, and the results from the two types of patterns were combined. Percent correct target categorization plotted as a function of SOA is shown in Figure 2. Performance in the absence of a nontarget was $99 \%$ correct. As expected, performance on RI trials (i.e., on trials in which the nontarget differed from the target and signaled a different response) reflects considerable interference. Performance was much below RC trials, when the nontarget differed from the target but signaled the same response. What is new with these results is the performance on neutral trials (i.e., trials on which a nontarget stimulus differed from the target but signaled neither a target nor a nontarget response). Performance on neutral trials appears to have been between $\mathrm{RI}$ and $\mathrm{RC}$ performance. A two-way repeated measures analysis of variance (ANOVA) revealed an overall effect of trial type $[F(3,15)=83.73, p<.0001]$. To see whether performance on neutral trials differed significantly from performance on RI trials, these data were analyzed separately. The RC and neutral data were also analyzed separately. Neutral pattern performance differed significantly from both $\mathrm{RC}[F(1,5)=28.99, p<.01]$ and $\mathrm{RI}$ $[F(1,5)=41.39, p<.01]$ performance. In other words, neutral performance was significantly below RC performance and significantly above RI performance. These results indicate that previous studies have underestimated the amount of interference due to masking.

Two aspects of these results support the view that nontarget patterns, at least those associated with responses, are processed to the point of evoking a response. First, performance on RC trials was considerably better than performance on neutral trials, presumably because the nontarget on RC trials evoked a correct response, whereas the nontarget on neutral trials evoked no response. Second, performance on RI trials was considerably below performance on neutral trials. In this case, the nontarget evoked

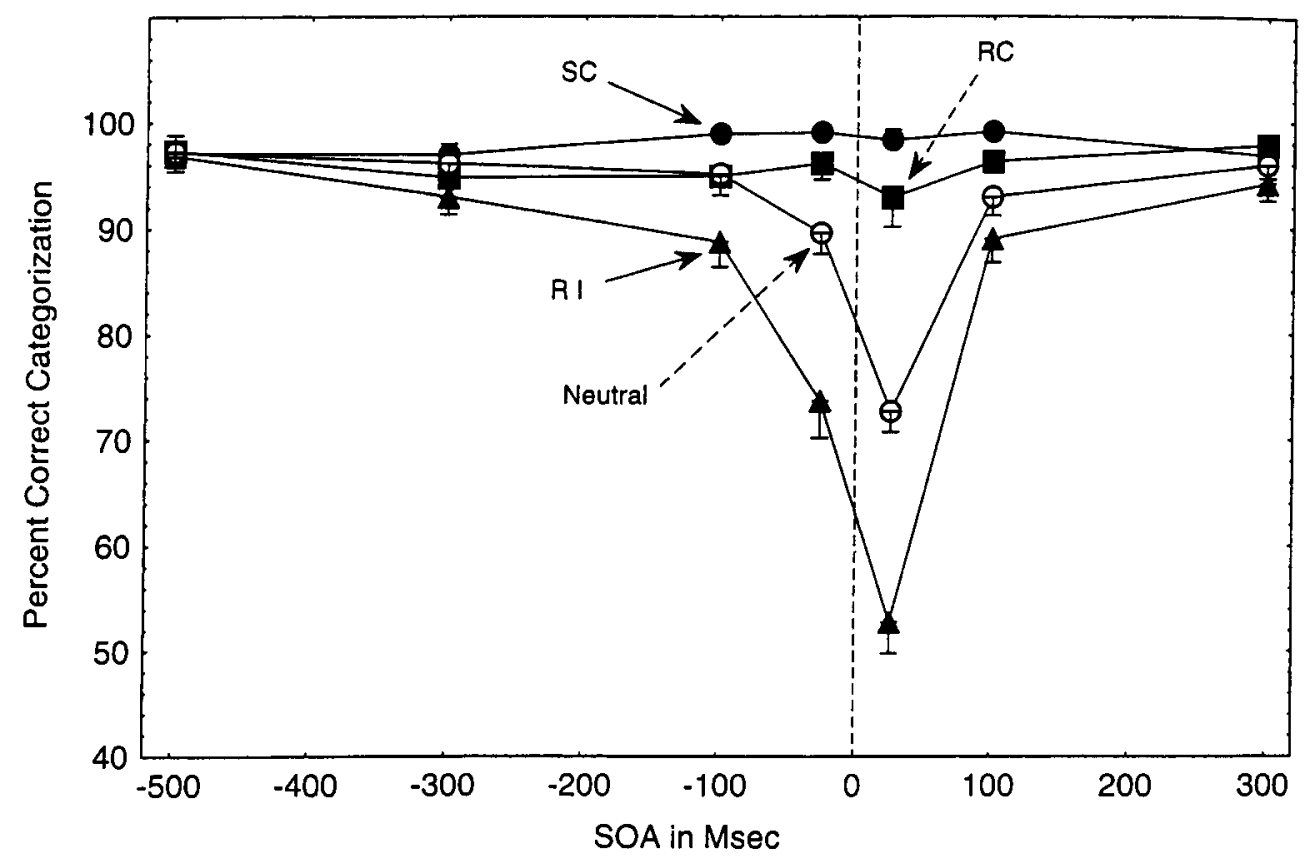

Figure 2. Percentages of correct categorizations as a function of the $\mathrm{SOA}$ between the onset of the target and nontarget. The results for four types of trials (SC, RC, RI, and neutral) are shown. Error bars represent 1 SEM. 


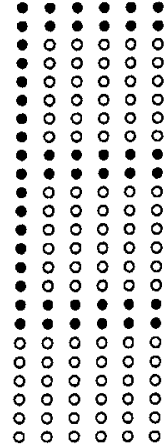

\section{Target}

Figure 3. Representations of the type of target pattern and neutral patterns used in Experiment 2.

an incorrect response and led to levels of performance well below those produced by the neutral nontargets.

\section{EXPERIMENT 2}

A possible problem with the 4-to-2 paradigm is that, with only two responses, subjects can respond correctly if they know which category was not presented. In Experiment 2, the number of target patterns was increased from four to 10 , and the number of response categories was increased from two to five. Also, more complex spatial patterns (letters) were used.

\section{Method}

Subjects. In Experiment 2, 9 subjects were tested: 7 women and 2 men.

Stimuli. The target patterns were 10 letters of the alphabet, "A, B, C, D, E, F, G, H, M, N," presented on the top 18 rows of the display. The dynamic masker and letter-like maskers were used as neutral patterns. The letter-like maskers were generated by selecting features of letters and combining them (Craig, 1982). The number of tactors averaged 46 for these letter-like maskers. The neutral pattern was selected randomly from a set of 10 of these patterns. Examples of the target and nontarget patterns are shown in Figure 3.

Procedure. The procedure was similar to that used in Experiment 1 . The subjects were trained to identify and place each of the 10 target patterns into one of five categories: "AB" in Category I, "CD" in Category II, and so forth. Of the total trials, $67 \%$ were RI, $8 \% \mathrm{RC}, 8 \% \mathrm{SC}$, and $16.5 \%$ neutral. The two neutral patterns, dynamic and letter-like maskers, were tested in separate blocks of trials. A single SOA of +52 msec was tested. The subjects completed 12 sessions, each consisting of 7 blocks of trials. Each block consisted of 40 trials.

\section{Results and Discussion}

The results are shown in Table 2. Target performance in the absence of a nontarget was $70 \%$ correct. The results with the letter-like neutral pattern and the dynamic neutral pattern were nearly identical $(t=-1.28, p>.05)$. The pattern of results was similar to that shown in Figure 2; SC and RC performances were similar to one another, with RI performance considerably lower and per- formance on neutral trials lying between performance on $\mathrm{RC}$ and RI trials. The effect of a neutral pattern was about the same whether the number of responses was two or five: Neutral patterns produce considerable masking, and it appears that performance on $\mathrm{RC}$ trials was enhanced because the subjects were responding with the nontarget. It is surprising that the letter-like neutral patterns did not produce more interference than the dynamic neutral patterns. One might expect that the features of the letter-like patterns would evoke target responses; and, with 10 targets and five categories, the likelihood is that these would be incorrect responses. In Experiments 3 and 4, we examined the effect of similarity of target and nontarget on target categorization.

\section{EXPERIMENT 3}

As noted, it was surprising that there was not more interference with the letter-like patterns used as neutral patterns in Experiment 2. The fact that the letter-like and dynamic neutral patterns produced similar amounts of interference in the perception of letters indicates that the letter-like patterns were not very effective at evoking responses. That is, they did not seem to "feel" like the target patterns.

In Experiment 3, we examined how the similarity of the neutral pattern affected target categorization. Rather than assume that a particular type of neutral pattern would be similar in general to the target patterns, we manipulated the probability that a neutral stimulus would evoke a response associated with the target pattern. Neutral patterns were selected that, on the basis of pretesting, had a reasonably high probability of being confused with at least one of the target patterns. In other words, the presentation of the neutral pattern would be expected to elicit a target response on some proportion of the trials.

\section{Method}

Subjects. In Experiment 3, 7 subjects were tested: 5 women and 2 men.

Procedure. The 4-to-2 paradigm was used. There were four target patterns and two neutral patterns, for a total of six patterns. All the patterns were letters of the alphabet. The letters were selected on the basis of previous measurements that showed that some of the target letters were likely to be confused with one another (Craig, 1979). The six letters were "A, D, F, K, O, X." Prior to the measurements with the 4-to-2 paradigm, the subjects were tested with the six patterns in an identification task. The patterns were presented one at a time for a duration of $26 \mathrm{msec}$. The subjects responded by means of a keypad marked with the six letters. Trial-bytrial feedback was provided. Seven subjects were tested for a total

Table 2

Percent Correct Responses for Letter Patterns

\begin{tabular}{ccccc}
\hline & \multicolumn{4}{c}{ Trial Type } \\
\cline { 2 - 5 } Neutral Condition & SC & RC & RI & Neutral \\
\hline Letter-like & 60 & 59 & 30 & 39 \\
Dynamic & 61 & 61 & 30 & 41 \\
\hline
\end{tabular}

Note-SC, stimulus compatible; RC, response compatible; RI, response incompatible. 


\begin{tabular}{|c|c|c|c|c|c|c|}
\hline & A & $D$ & $F$ & $\mathrm{~K}$ & 0 & $x$ \\
\hline A & $95.0 \%$ & $0.4 \%$ & $0.2 \%$ & $1.5 \%$ & $1.3 \%$ & $1.7 \%$ \\
\hline & $0.0 \%$ & $58.3 \%$ & $0.2 \%$ & $0.2 \%$ & $41.2 \%$ & $0.2 \%$ \\
\hline$F$ & $0.8 \%$ & $0.2 \%$ & $97.3 \%$ & $1.8 \%$ & $0.0 \%$ & $0.0 \%$ \\
\hline & $2.6 \%$ & $0.4 \%$ & $2.4 \%$ & $72.2 \%$ & $0.4 \%$ & $22.2 \%$ \\
\hline & $3.2 \%$ & $39.8 \%$ & $0.5 \%$ & $0.5 \%$ & $55.8 \%$ & $0.2 \%$ \\
\hline & $3.1 \%$ & $0.0 \%$ & $0.5 \%$ & $9.2 \%$ & $0.0 \%$ & $87.1 \%$ \\
\hline
\end{tabular}

Figure 4. A confusion matrix showing the percentages of subjects' responses to the six letters used as patterns in Experiment 3.

of 3,300 trials. A confusion matrix was constructed and is shown in Figure 4. On the basis of this confusion matrix, letters " $A, O$ " were assigned to Category $I$, and letters " $F$, $X$ " were assigned to Category II. " $D$ " was selected as one of the neutral patterns because the subjects often responded "O" when presented with it. Similarly, " $K$ " was selected as the second neutral pattern because the subjects often responded " $X$ " when presented with it.

In the main portion of the experiment, the procedure was similar to that used in Experiment 1. There were eight blocks of trials per testing session, one block for each of the seven SOAs and one block with the target pattern presented by itself. The patterns were presented for $26 \mathrm{msec}$. The approximate distribution of trial types was as follows: $16.5 \% \mathrm{SC}, 16.5 \% \mathrm{RC}, 33 \% \mathrm{RI}$, and $33 \%$ neutral. Be- cause we wanted to analyze the results in terms of the four types of target patterns and the two types of neutral patterns, a large number of testing sessions (28) were conducted.

\section{Results and Discussion}

Figure 5 shows overall categorization performance as a function of SOA for the first set of measurements. Performance in the absence of a nontarget was $97 \%$ correct. As in Experiment 1, performance with neutral patterns was between RI and RC performance. More important than the overall results are the results with the individual neutral patterns and target patterns.

If neutral pattern " $D$ " is presented with one of the patterns from Category I, either " $A$ " or " $O$," then performance levels might be expected to be more similar to RC levels, because " $D$ " is confused with " $O$;" however, when neutral pattern " $K$ " is presented with either " $A$ " or "O," performance levels might be similar to RI levels because it is associated with " $\mathrm{X}$," a member of response Category II. The results for each of the four target patterns are shown in the four panels of Figure 6 . The two functions presented in each panel show separately the results with the two different neutral patterns. It is clear that the two neutral patterns had a large differential effect on the target patterns. This effect was such that when the target pattern was "A" (Figure 6A) and the neutral pattern was "D," performance was as high as it was on RC trials (Figure 5); whereas, when the neutral pattern was "K," performance was as low as it was on RI trials (Figure 5). The results with the remaining targets were similar (Figures $6 \mathrm{~B}$,

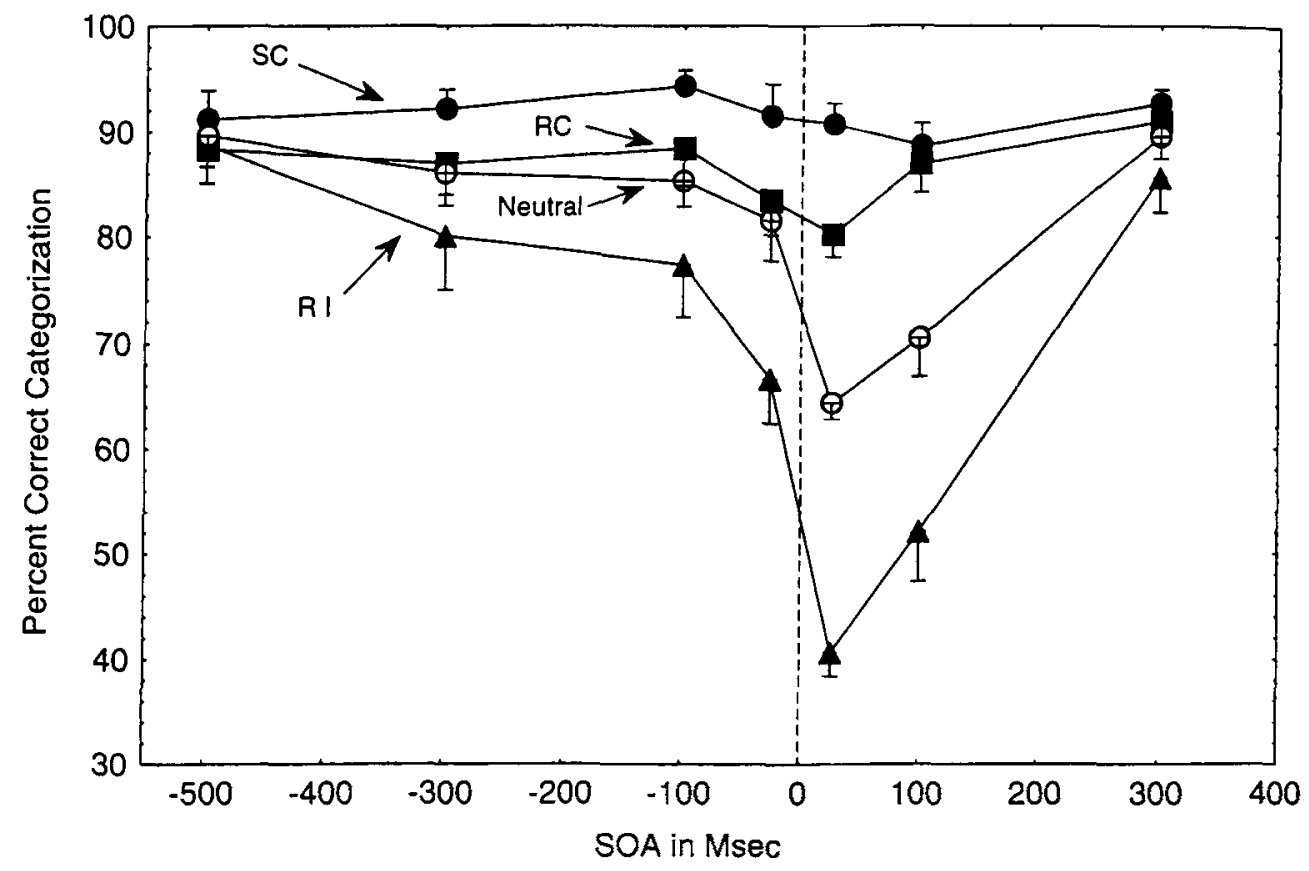

Figure 5. Percentages of correct categorizations as a function of SOA for letter patterns in Experiment 3. The results for four types of trials (SC, RC, RI, and neutral) are shown. Error bars represent $1 S E M$. 

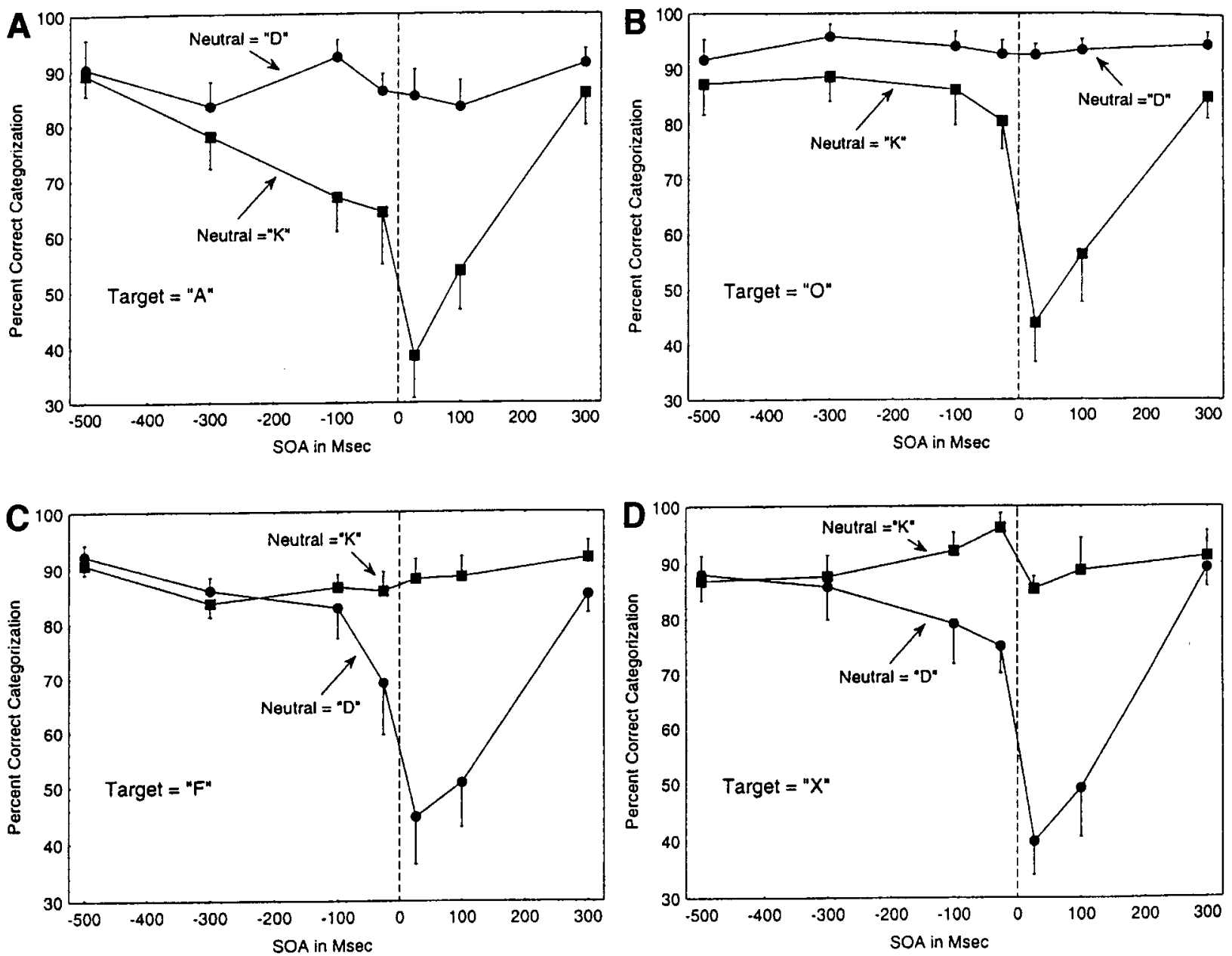

Figure 6. The results from the neutral trials in Figure 5 replotted to show performance with the two different neutral patterns, " $D$ " and "K." Each panel represents the results with a different target pattern. Error bars represent 1 SEM.

$6 \mathrm{C}$, and $6 \mathrm{D})$. When the target was paired with a neutral pattern that was similar to patterns in the category to which it belonged, performance levels were high. When paired with a neutral pattern that was similar to a pattern in the opposite category, performance levels were low. When a neutral pattern was confusable with a pattern associated with a particular response category, the neutral pattern acted as an RC pattern for that category and an RI pattern for the opposite category.

Another way to think about the neutral patterns " $D$ " and " $\mathrm{K}$ " is that they were not actually neutral patterns, because they had some associative value with the target patterns. What is surprising is that their effectiveness was indistinguishable from a target pattern, even though in the case of " $\mathrm{K}$ " it was confused with "X" on only $22 \%$ of the trials (Figure 4). The reason for this effectiveness may be that the confusability among patterns was originally determined by presenting the patterns in isolation. It is likely that presenting the patterns in pairs increases the confusability between the patterns. This otservation is consistent with the view that there is mutual masking between the target and the nontarget.

\section{EXPERIMENT 4}

In Experiment 4, we examined another way to vary the similarity between the nontarget and target patterns and the effect of the similarity on target processing. Neutral patterns were selected that were composites of two of the target patterns. In this situation, the neutral pattern presented by itself would be unlikely to be confused with one of the target patterns presented by itself, but it might well be confused with two patterns presented in close temporal proximity. Previous results showed that subjects often responded to two patterns presented close together in time as though a single composite had been presented (Craig, 1998; Evans \& Craig, 1986).

\section{Method}

Subjects. In Experiment 4, 5 subjects were tested: 3 women and 2 men.

Procedure. Two neutral patterns were designed to be composites of two of the target patterns. The target patterns were the same as those used in Experiment 1 (Figure 1). The two neutral patterns are shown in Figure 7. The first neutral pattern, Pattern A, is a composite of Patterns 1 and 2 (Figure 1), and the second pattern, Pattern B, 


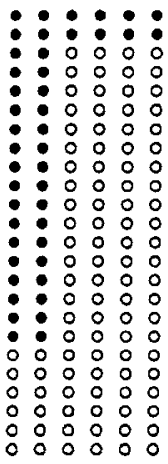

A

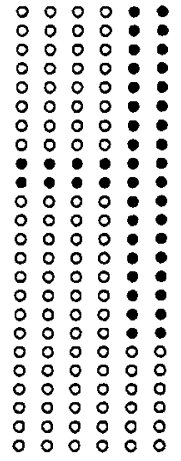

B
Figure 7. Representations of the two neutral patterns used in Experiment 4. The patterns are composites of the target patterns shown in Figure 1.

is a composite of Patterns 3 and 4 . The same procedure was followed as in earlier 4-to-2 measurements, with the exception that pattern duration was reduced to $13 \mathrm{msec}$. The overall percentages of SC, RC, RI, and neutral patterns were the same as Experiment 1 . Each of the two neutral composite patterns was presented on about half of the neutral trials. The subjects were tested for 11 sessions.

\section{Results and Discussion}

The data, presented in Figure 8, show the overall results obtained with the two composite neutrals. Performance in the absence of a nontarget was $99 \%$ correct. These results look very much like the previous functions, with neutral performance falling somewhere between
$\mathrm{RC}$ and RI performance. The data were analyzed separately for the two different neutral patterns and for the categories with which they are associated. The data are replotted in Figure 9 to show the effect that a neutral pattern had on performance when the target was selected from the category with which the neutral pattern was associated (the neutral pattern was a composite of the two targets in that category) or selected from the opposite category. For example, in Figure 9A, neutral A was the composite of the two patterns in Category I, and neutral B was the composite of the two patterns in Category II. The pattern of results was quite different from that shown in Figure 8. When the neutral was a composite of two patterns from a different category than the target, the results were almost identical to those from RI trials. When the neutral pattern was a composite of two patterns from the same category as the target, the results were almost identical to those from RC trials. In short, the composite patterns produced effects very much like the effects of target patterns.

The composite patterns, unlike the neutral patterns used in Experiment 3, are unlikely to be confused with a target pattern presented by itself. The composites are very likely to be confused with a pair of target patterns presented simultaneously or nearly so. On a neutral trial, the subjects received a target plus a neutral pattern that was, in fact, composed of two target patterns - in effect, three patterns. The subjects responded not as though they felt three patterns but as though they felt only two patterns. The results might lead one to conclude that the target pattern was eliminated by the composite. Results from other studies indicate that this is unlikely to be the case (Craig,

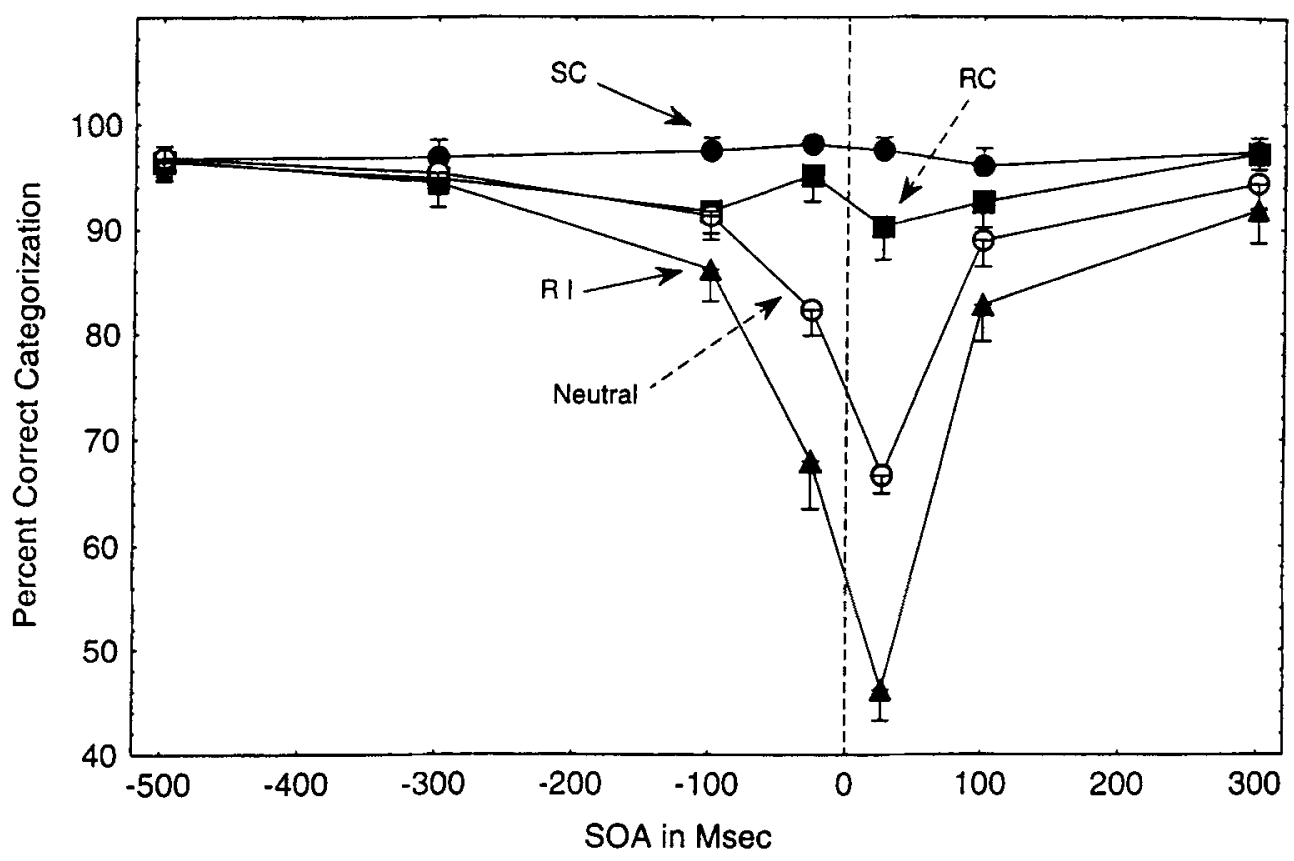

Figure 8. Percentages of correct categorizations as a function of SOA for Experiment 4. The results for four types of trials (SC, RC, RI, and neutral) are shown. Error bars represent 1 SEM. 

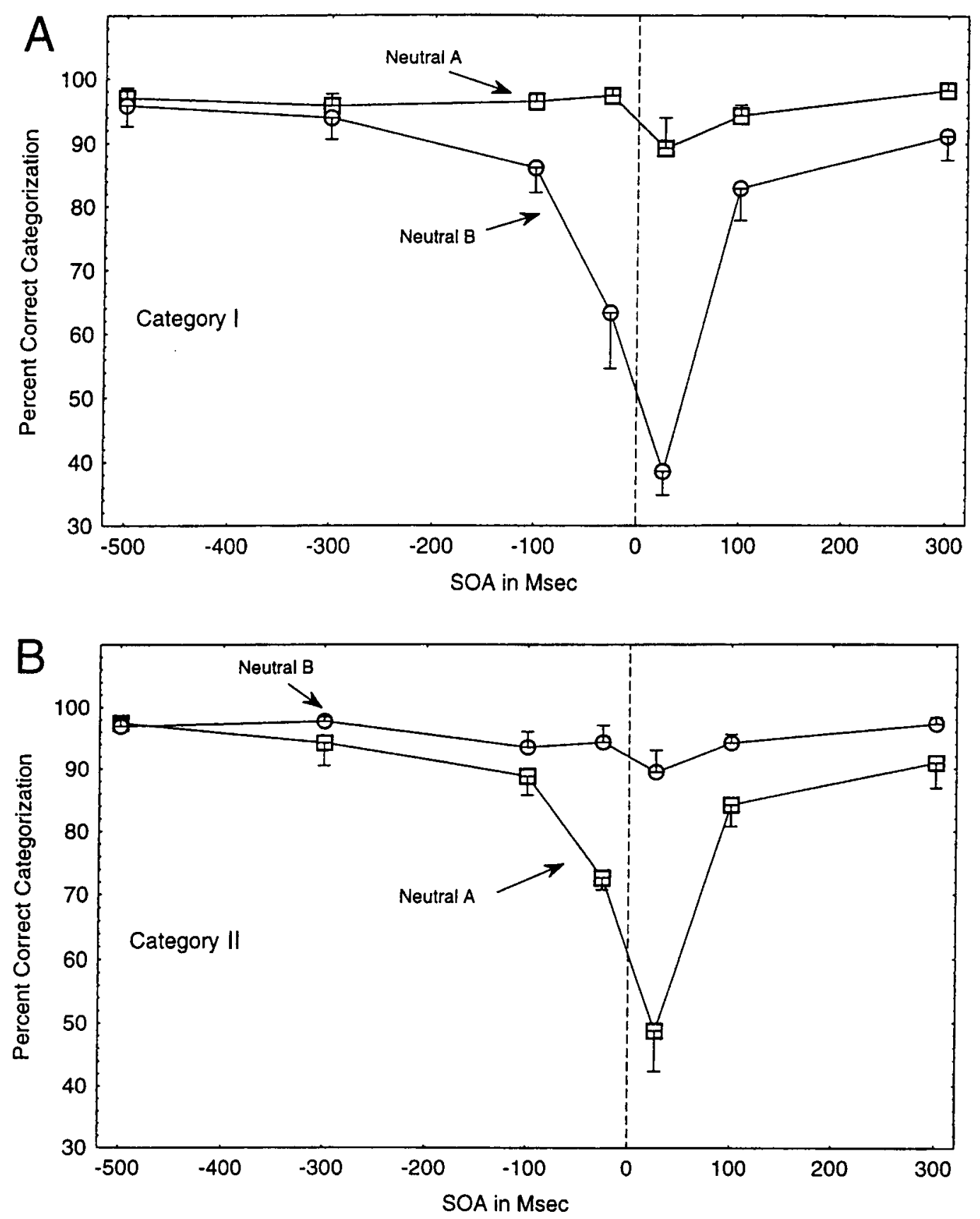

Figure 9. Data from Figure 8 replotted to show the results on Category I patterns and Category II patterns by the two different neutral patterns. (A) The results with the patterns from Category $I$. The function marked "Neutral A" represents the results from Neutral Pattern A (Figure 7). The function marked "Neutral B" represents the results from Neutral Pattern B (Figure 7). (B) The results with patterns from Category II. Error bars represent 1 SEM.

1995). What is likely is that the subjects chose between two patterns: the composite (a relatively clear representation that resulted from two target patterns presented in close temporal contiguity) and a less clear representation of a target pattern. The perceived intensity of the composite may have even been enhanced by the presentation of the target pattern because the two had a number of features in common.

\section{GENERAL DISCUSSION}

The main conclusions from the present study are the following: (1) Previous studies examining the effects of response competition and masking have likely underestimated the amount of interference due to masking. (2) Nontarget patterns are very likely to be responded to as though they were targets. If the nontarget is associated 
with a correct response, levels of performance will increase. If the nontarget is associated with an incorrect response, levels of performance will decrease. (3) The greater the similarity between the target and nontarget, the more likely subjects are to respond with the nontarget.

Generally, response competition has been viewed as a process that interferes with correct responding, either increasing the number of errors or lengthening reaction times. The present results indicate that response competition also increases correct responses, when both the target and the nontarget are associated with the same response. One might question whether the term response competition is appropriate when the two patterns evoke the same response; but, whether the two patterns are associated with the same or different responses, the process is the same: Both target and nontarget patterns are processed to the point of evoking responses.

How do the present results apply to processing spatial patterns in reading with the Optacon or reading braille or in the haptic identification of an object? The results indicate that target patterns are subject to more masking than previously estimated: The representation of the target pattern is subject to more interference at an early stage of processing. A conclusion from a previous study was that context cues could be used to help identify a pattern if the main source of interference were response competition (Craig, 1996). Context cues may be less useful if the interference is the result of masking. Also, the majority of the interference takes place when the onsets of the two patterns are within approximately $100 \mathrm{msec}$ of one another. This temporal window may impose a limitation on reading rates with the Optacon and with braille. Although unusual, Optacon reading rates of up to 100 words per minute have been reported (Craig, 1977). Assuming 5.5 characters per word with a space at the end of each word, the SOA between successive letters would be about $150 \mathrm{msec}$. This temporal separation may be the point when both masking, as evidenced by the neutral functions, and response competition, as evidenced by the RI functions, reduce pattern recognition to levels that render reading very difficult.

The present results suggest that it is possible to separate the effects of masking from the effects of response competition without using a categorization task, such as the 4-to-2 paradigm, and RC trials. Interpreting the results from RC trials is difficult because performance may reflect both masking and response competition. If the measure of performance is percent correct rather than reaction times, then the use of neutral patterns eliminates the need for RC trials. This assumes that suitable and appropriate neutral patterns are used. The new paradigm would use an identification task. Nontargets would consist of both other possible targets (an RI trial) and neutral patterns. This paradigm has some advantages over the 4to-2 paradigm. It makes possible a clearer interpretation of subjects' responses. In the 4-to-2 paradigm, when a subject makes a category response, it is unclear which one of several patterns the subject in fact perceives. Interpreting subjects' responses is easier when each target is associated with a unique response. Also, a situation in which a person is trying to identify, rather than categorize, a tactile pattern is more easily generalized to other tactile tasks than a situation in which several different patterns have the same response associated with them.

\section{REFERENCES}

Bliss, J. C., Katcher, M. H., Rogers, C. H., \& Shepard, R. P. (1970). Optical-to-tactile image conversion for the blind. IEEE Transactions on Man-Machine Systems, MMS-11, 58-64.

Cholewiak, R. W., \& Collins, A. A. (1988). Vibrotactile pattern masking with static and dynamic tactile noise [Abstract]. Bulletin of the Psychonomic Society, 26, 487.

Cholewiak, R. W., \& Col.Lins, A. A. (1997). Individual differences in the vibrotactile perception of a "simple" pattern set. Perception \& Psychophysics, 59, 850-866.

Cholewiak, R. W., \& Craig, J. C. (1984). Vibrotactile pattern recognition and discrimination at several body sites. Perception \& Psychophysics, 35, 503-514.

CRAIG, J. C. (1977). Vibrotactile pattern perception: Extraordinary observers. Science, 196, 450-452.

CraIG, J. C. (1979). A confusion matrix for tactually presented letters. Perception \& Psychophysics, 26, 409-411.

CraIG, J. C. (1980). Modes of vibrotactile pattern perception. Journal of Experimental Psychology: Human Perception \& Performance, 6, 151-166.

Craig, J. C. (1982). Vibrotactile masking: A comparison of energy and pattern maskers. Perception \& Psychophysics, 31, 523-529.

Craig, J. C. (1989). Interference in localizing tactile stimuli. Perception \& Psychophysics, 45, 343-355.

Craig, J. C. (1995). Vibrotactile masking: The role of response competition. Perception \& Psychophysics, 57, 1190-1200.

CraIG, J. C. (1996). Interference in identifying tactile patterns: Response competition and temporal integration. Somatosensory \& Motor Research, 13, 199-213.

Craig, J. C. (1998). Vibrotactile pattern isolation/integration. Perception \& Psychophysics, 60, 888-899.

Craig, J. C., \& Evans, P. M. (1995). Tactile selective attention and temporal masking. Perception \& Psychophysics, 57, 511-518.

Craig, J. C., \& Qian, X. (1997). Tactile pattern perception by two fingers: Temporal interference and response competition. Perception \& Psychophysics, 59, 252-265.

Eriksen, C. W., \& Hoffman, J. E. (1973). The extent of processing noise elements during selective encoding from visual displays. Perception \& Psychophysics, 14, 155-160.

Evans, P. M. (1987). Vibrotactile masking: Temporal integration, persistence, and strengths of representations. Perception \& Psychophysics, 42, 515-525.

Evans, P. M., \& Craig, J. C. (1986). Temporal integration and vibrotactile backward masking. Journal of Experimental Psychology: Human Perception \& Performance, 12, 160-168.

Evans, P. M., \& Craig, J. C. (1992). Response competition: A major source of interference in a tactile identification task. Perception \& Psychophysics, 51, 199-206.

FoulKe, E. (1991). Braille. In M. A. Heller \& W. Schiff(Eds.), The psychology of touch (pp. 219-233). Hillsdale, NJ: Erlbaum.

HiLL, J. W. (1974). Limited field of view in reading lettershapes with the fingers. In F. A. Geldard (Ed.), Cutaneous communication systems and devices (pp. 95-105). Austin, TX: Psychonomic Society.

Lederman, S. J., \& KLATZKY, R. L. (1993). Extracting object properties through haptic exploration. Acta Psychologica, 84, 29-40.

LedERMAN, S. J., \& KLATZKY, R. L. (1997). Relative availability of sur- 
face and object properties during early haptic processing. Journal of Experimental Psychology: Human Perception \& Performance, 23, 1-28.

MAHAR, D. P., \& MACKENZIE, B. D. (1993). Masking, information integration, and tactile pattern perception: A comparison of the isolation and integration hypothesis. Perception, 22, 483-496.

Sathian, K., \& Burton, H. (1991). The role of spatially selective attention in the tactile perception of texture. Perception \& Psychophysics, 50, 237-248.

SCHINDLER, U., \& KNAPP, A. (1976). Ursachen der gegenseitigen Verdeckung von taktil dargebotenen Buchstaben: Unterbrechung,
Summation oder Verzogerung? [Sources of mutual masking of tactile presented letters: Interruption, summation, or delay?] Psychological Research, 38, 303-318.

Whang, K. C., Burton, H., \& Shulman, G. L. (1991). Selective attention in vibrotactile tasks: Detecting the presence and absence of amplitude change. Perception \& Psychophysics, 50, 157-165.

(Manuscript received September 8, 1998; revision accepted for publication April 15, 1999.) 